\title{
A NOVA-LIKE RED VARIABLE IN M31
}

\author{
Jeremy Mould, Judith Cohen, James R. Graham, Don Hamilton, Keith Matthews, Alain Picard, \\ Neill Reid, MaARTen Schmidt, Tom Soifer, AND Christine Wilson \\ Palomar Observatory, California Institute of Technology \\ R. MiCHAEL RiCH
Columbia University \\ AND \\ JAMES GUNN \\ Princeton University Observatory \\ Received 1989 November 14 ; accepted 1990 February 7
}

\begin{abstract}
A late-type supergiant which appeared in the bulge of M31 in the last observing season faded 3 bolometric magnitudes in 100 days and is now no longer detectable. We suggest the object was a nova of an unusual type.
\end{abstract}

Subject headings: galaxies: individual (M31) - stars: late-type — stars: novae

\section{INTRODUCTION}

On 1988 September 2 a 15 th mag red variable star was detected 3' from the center of M31 with a spectral type M0 Ie (Rich et al. 1989). In this Letter we describe the subsequent decline of this star, which remained a simple $\mathbf{M}$ supergiant in the appearance of its spectrum, but whose light curve resembles that of a nova. In $\S$ II the bolometric evolution is detailed; $\S$ III describes the behavior of the spectrum; and in $\S$ IV we discuss what kind of system could have erupted in this way.

\section{PHOTOMETRY}

Initially the colors of this star were those of a slightly reddened early M supergiant (Rich et al. 1989). In the ensuing weeks observations at Palomar Observatory were carried out in the $I$ band with the $1.5 \mathrm{~m}$ telescope, and with an infrared camera attached to the Cassegrain focus of the $5 \mathrm{~m}$ Hale telescope. The procedure for photometry from the infrared images is detailed by Mould et al. (1989). All available photometry is contained in Table 1.

The decline in luminosity of the star is shown in Figure 1. From an initial luminosity of $8 \times 10^{5} L_{\odot}$ (Rich et al. 1989) it faded to $6 \times 10^{4} L_{\odot}$ in 70 days and fell below $10^{4} L_{\odot}$ in 290 days. Luminosities from JD 2,447,450 onward were evaluated by integrating the energy distribution of Figure 2 and extrapolating with a blackbody normalized at $3.6 \mu \mathrm{m}$. A blackbody of $2000 \mathrm{~K}$ is a reasonable fit to the near-infrared data at JD $2,447,460$ and is used to extrapolate the energy distribution beyond $3.6 \mu \mathrm{m}$. The adopted value of $2000 \mathrm{~K}$ does not significantly affect the calculation of an integrated luminosity. The adopted temperature at JD 2,447,485 was $1050 \mathrm{~K}$ and is important in the luminosity calculation. This temperature is somewhat cooler than the near-infrared data require. It is adopted because a value of the order of $1000 \mathrm{~K}$ is typical for the infrared development of novae (Gehrz 1988). A distance modulus of 24.2 was assumed for M31 (Mould 1988).

\section{SPECTROSCOPY}

The spectrum of the star on JD 2,447,437.93 was obtained with the Double Spectrograph (Oke and Gunn 1982) and is reproduced in Figure 3. A further spectrum was secured on JD
2,447,466.5 with 4SHOOTER (Gunn et al. 1987) and appears in Figure 4. Over this period the spectrum changed to a later $M$ type. TiO bands dominate these spectra. The only emission lines present are Balmer lines. Quantitative details are contained in Table 2. The quoted velocities are heliocentric and represent a blueshift relative to the systemic velocity of M31 of $\approx 150 \mathrm{~km} \mathrm{~s}^{-1}$.

\section{A NOVA OF AN UNUSUAL TYPE}

Rich et al. (1989) refer to this star as a red variable star but describe its behavior in terms of an "outburst." This is a good summary of the object's peculiarity, as can be seen from the subsequent discussion.

The spectroscopic development summarized in Table 2 most resembles the behavior of Mira variables. There are two difficulties with the Mira interpretation, however. The large amplitude which has become evident in recent observations is at the limit of what is seen in OH-IR variables (Engels et al. 1982). Furthermore, the light curves of those OH-IR stars which have amplitudes at $2.2 \mu \mathrm{m}$ reaching $4 \mathrm{mag}$ are near-sinusoidal in flux, not in magnitude. Therefore the mean luminosity of star $\mathrm{RV}$ as a Mira clearly exceeds the AGB limit ( $M_{\text {bol }} \sim-7 \mathrm{mag}$ ). Supergiant long-period variables (such as VX Sgr) exist but do not have the large amplitude we see here.

The high-outburst luminosity of star RV is more suggestive of a nova, however. Three of the four criteria for membership in the nova class listed by Gallagher and Starrfield (1978) are clearly satisfied by this object (luminosity change, time scale, and ejection velocity). The striking discrepancy is the lack of any coronal or nebular phase of gaseous emission. In addition, a quantitative discrepancy with other novae which have reached $M_{\text {bol }}=-10 \mathrm{mag}$ at maximum light is the order-ofmagnitude longer fading time of star RV. It seems unlikely that the standard nova model of an outburst near the surface of a white dwarf accreting from a main-sequence star is appropriate to this case. To account for the properties of this object, one might consider some other scenarios, for example a model in which a nova outburst occurred on a compact companion within a common red giant envelope. 


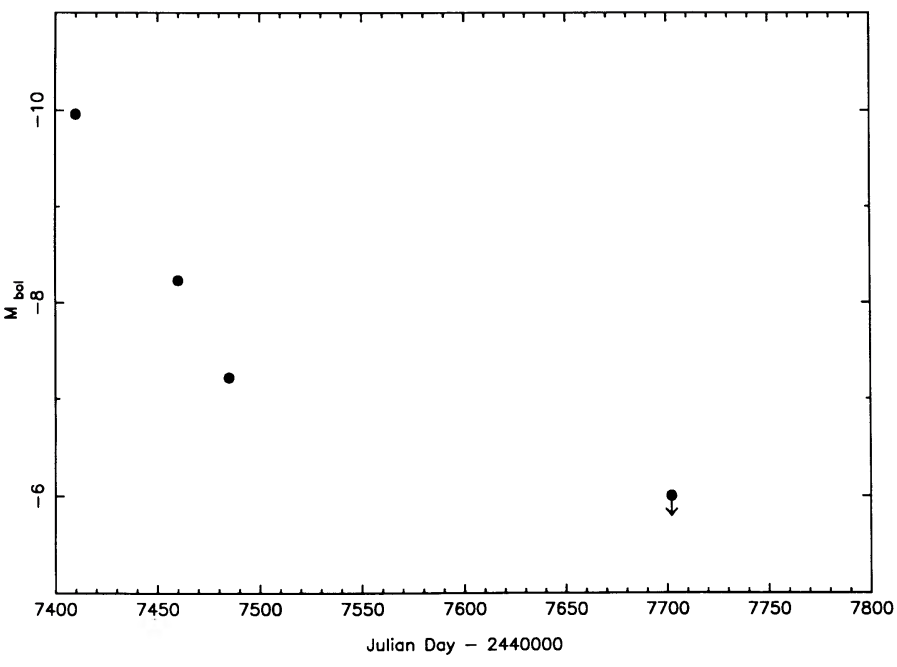

FIG. 1.-Light curve in bolometric magnitudes

Serious modeling of such an outburst is beyond the scope of this Letter, but to stimulate such calculations, we offer the following outline of the relevant physical parameters. Under the assumption that the star was detected within a short time of the outburst, we calculate the total energy radiated in the first hundred days to be $10^{46}$ ergs. Now a model is required to tell us approximately how the energy of the explosion divides itself between kinetic energy of expansion, radiation, and the

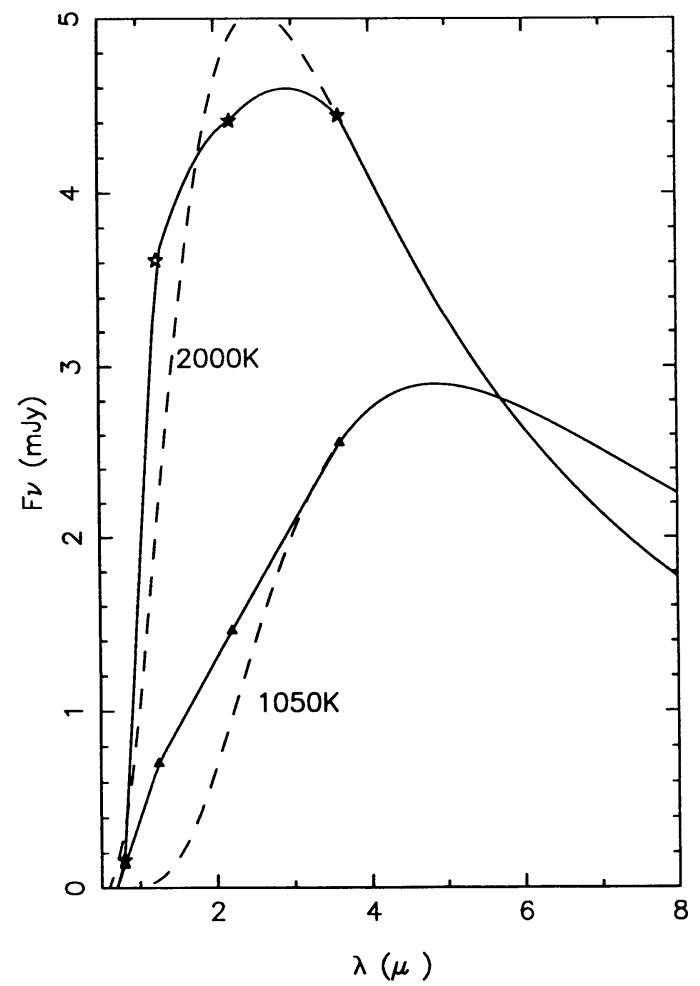

FIG. 2.-Energy distribution at JD 2,447,460 shown by the solid line connecting the star symbols. The dashed line is a $2000 \mathrm{~K}$ blackbody fit. The energy distribution at JD 2,447,485 is the solid line connecting the triangles. The associated dashed line is a $1050 \mathrm{~K}$ blackbody.
TABLE 1

PHOTOMETRY

\begin{tabular}{cccccccc}
\hline \hline J.D. $^{2}$ & $\boldsymbol{I}^{\mathrm{b}}$ & $\boldsymbol{J}$ & $\boldsymbol{H}$ & $K$ & $L$ & $M_{\text {bol }}$ & Observer \\
\hline $7409.9 \ldots \ldots$ & $14.9^{\mathrm{c}}$ & $\ldots$ & $\ldots$ & $\ldots$ & $\ldots$ & -9.8 & RMR $^{\mathrm{d}}$ \\
$7425.9 \ldots \ldots$ & $\ldots$ & 12.87 & 12.23 & 12.01 & $\ldots$ & -9.96 & RMR \\
$7452.9 \ldots \ldots$ & 17.2 & $\ldots$ & $\ldots$ & $\ldots$ & $\ldots$ & $\ldots$ & CDW \\
$7459.7 \ldots \ldots$ & $18.4^{\mathrm{e}}$ & 14.1 & $\ldots$ & 12.8 & 12.0 & -8.2 & JRM \\
$7471.8 \ldots \ldots$ & $>18.6$ & $\ldots$ & $\ldots$ & $\ldots$ & $\ldots$ & $\ldots$ & AP \\
$7484.6 \ldots \ldots$ & $\ldots$ & 15.87 & 14.77 & 14.0 & 12.6 & -7.2 & BTS \\
$7702.7 \ldots \ldots$ & $\ldots$ & $>16.8$ & $\ldots$ & $>15.6$ & $\ldots$ & $\ldots$ & JGC \\
$7710.8 \ldots \ldots$ & $>19.3$ & $\ldots$ & $\ldots$ & $\ldots$ & $\ldots$ & $\ldots$ & JRM \\
\hline
\end{tabular}

Julian date $-2,444,0000$.

${ }^{b}$ Cousins system.

c Gunn system.

${ }^{d}$ Rich et al. 1989.

e Actual date of $i$ photometry: JD 2,444,7458.9.

work done to expand the envelope. However, lacking such a model, if we naively equate the kinetic energy in the moving shell to the radiated energy (neglecting the adiabatic losses which may be dominant), and assume an expansion velocity of $100-500 \mathrm{~km} \mathrm{~s}^{-1}$, the mass of the shell is $10^{-1}$ to $10^{-3} M_{\odot}$. The expansion velocity is uncertain by the velocity dispersion of stars in the bulge of M31, but since it is at the low end of the range for novae, whereas the energy output is at the high end of the range, a large envelope mass seems indicated. At maximum light, the radius of the $4000 \mathrm{~K}$ photosphere is $\sim 2000 R_{\odot}$. After 70 days we see the radiation from a $1000 \mathrm{~K}$ dust shell with $R \sim 8000 R_{\odot}$. The shell remains optically thick through this expansion. A mass of $\sim 10^{-2} M_{\odot}$ is more than is normally

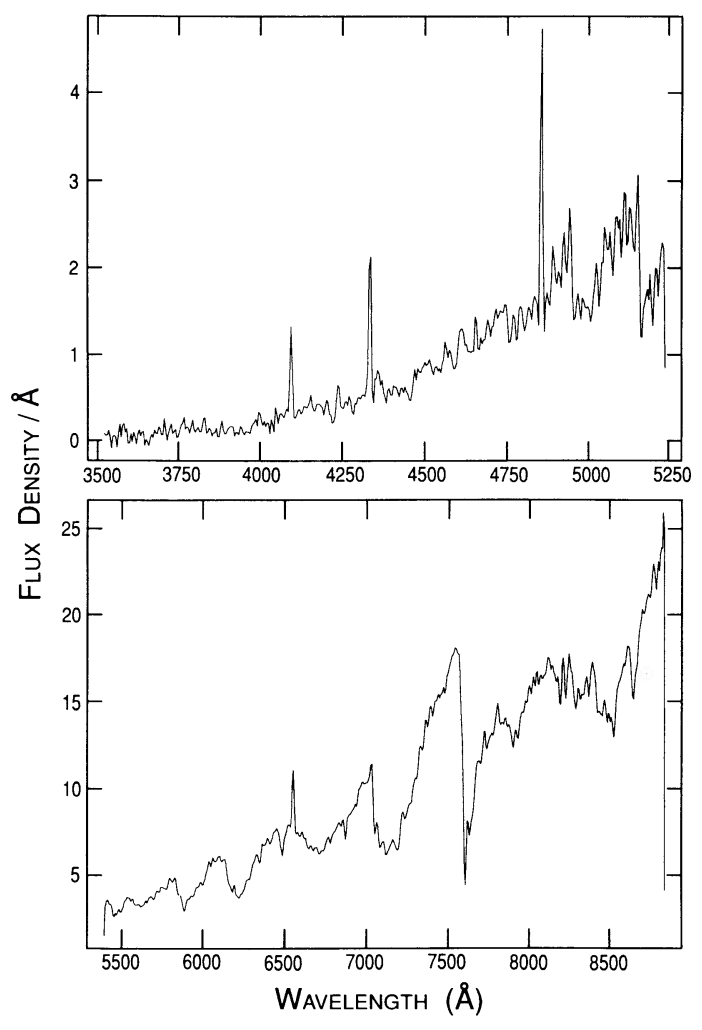

Fig. 3.-Spectrum on JD $2,447,438$ 


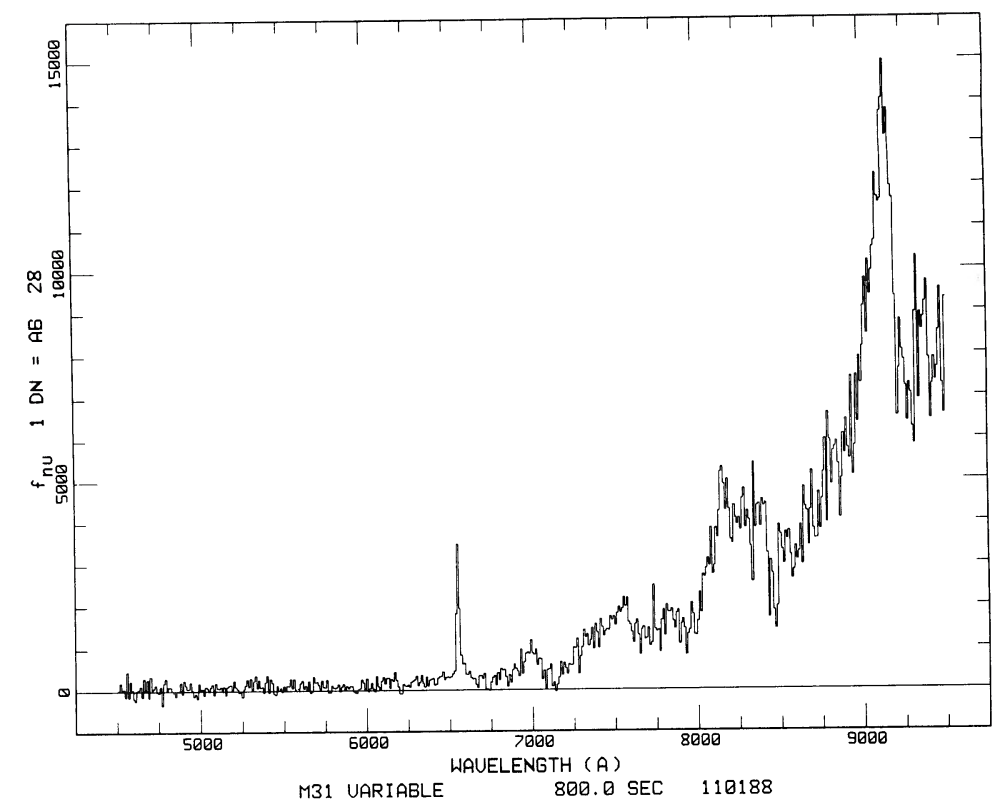

Fig. 4. - Spectrum on JD 2,447,466.5

TABLE 2

SPECTROSCOPY

\begin{tabular}{lccccc}
\hline \hline $\begin{array}{c}\text { J.D. } \\
2,440,000+\end{array}$ & $\begin{array}{c}\text { Spectral } \\
\text { Type }\end{array}$ & $\begin{array}{c}\text { Velocity } \\
\left(\mathrm{km} \mathrm{s}^{-1}\right)\end{array}$ & $\begin{array}{c}\mathrm{H} \alpha \\
\left(\times 10^{-15} \mathrm{ergs}\right. \\
\left.\mathrm{cm}^{-2} \mathrm{~s}^{-1}\right)\end{array}$ & $\begin{array}{c}\mathrm{H} \beta \\
\left(\times 10^{-15} \mathrm{ergs}\right. \\
\left.\mathrm{cm}^{-2} \mathrm{~s}^{-1}\right)\end{array}$ & Observer \\
\hline $7409.5 \ldots \ldots$ & M0 Ie & $-610 \pm 100$ & 3.1 & $\ldots$ & RMR \\
$7409.9 \ldots \ldots$ & $\ldots$ & $-220 \pm 60$ & $\ldots$ & $\ldots$ & RMR \\
$7437.9 \ldots \ldots$ & M3 Ie & $-435 \pm 20$ & 5.0 & 2.8 & DH \\
$7466.9 \ldots \ldots$ & M6 Ie & $-560 \pm 100$ & 1.5 & $\ldots$ & DPS \\
\hline
\end{tabular}

${ }^{\text {a }}$ Heliocentric radial velocity.

ejected in a nova explosion but may be what we need in order to avoid a nebular phase.

Finally, we emphasize that star RV does not comfortably fit into the standard scenarios for eruptive events on white dwarfs. It evolves slowly, has highly super-Eddington luminosity, and is ejecting a lot of mass. The spectrum is too cool for any nova that we can safely associate with a such a powerful thermonu- clear runaway on a white dwarf. Alternative models for this unique event should be explored.

We are grateful to Don Schneider for reducing the spectrum shown in Figure 4 and to Jay Gallagher for reviewing the manuscript. Partial support for this work was provided by NSF grants AST 86-13059 and 87-21705 to Caltech.

\section{REFERENCES}

Elias, J., Frogel, J., Matthews, K., and Neugebauer, G. 1981, A.J., 87, 1029.

Engels, D. Kreysa, E., Schultz, G. and Sherwood, W. 1982, Astr. Ap., 124, 123. Gallagher, J. and Starrfield, S. 1978, Ann. Rev. Astr. Ap., 16, 171

Gehrz, R. 1988, Ann. Rev. Astr. Ap., 26, 377.

Gunn, J., et al. 1987, Opt. Eng., 26, 779.

Mould, J. 1988, in The Extragalactic Distance Scale, ed. C. Pritchet and S. van den Bergh (ASP Conf. Ser., 4), p. 32
Mould, J., Graham, J., Matthews, K., Phinney, E. S., and Soifer, B. T. 1989, Ap. J.(Letters), 339, L21.

Oke, J. B., and Gunn, J. 1982, Pub. A.S.P., 94, 586

Rich, R., Mould, J., Picard, A., Frogel, J., and Davies, R. 1989, Ap. J. (Letters), 341, L51.

Judith Cohen, Jeremy Mould, Alain Picard, MaArten Schmidt, and Christine Wilson: Caltech, 105-24, Pasadena, CA 91125

James Graham, Keith Matthews, and Tom Soifer: Caltech, 320-47, Pasadena, CA 91125

JAMES GuNN: Peyton Hall, Princeton University, Princeton NJ 08544

R. Michael Rich: Department of Astronomy, Columbia University, New York, NY 10027 